\title{
Musicoterapia en Polimicrogiria. Una propuesta de intervención
}

\author{
Raquel Ortega Ilarregui ${ }^{1}$ \\ Recibido: 02/06/2020
}

Aceptado: 09/06/2020

RESUMEN Este trabajo propone un protocolo de intervención musicoterapéutica para pacientes con polimicrogiria, una enfermedad rara que cursa con un incorrecto patrón de pliegues en la corteza cerebral. Tras realizar una revisión sobre esta patología, se han analizado las necesidades del colectivo abordables desde la musicoterapia estableciéndose unos objetivos terapéuticos. Según estos, se han diseñado las actividades. El protocolo diseñado, se ha testado en una paciente con polimicrogiria (13 meses de edad). Los resultados han sido favorables, ya que las sesiones han potenciado su atención mantenida, disminuido su frecuencia cardiaca, y estimulado su motricidad. También se valoraron los conocimientos previos sobre musicoterapia de los padres a través de un cuestionario, concluyéndose que esta terapia no está considerada oficialmente dentro de las terapias complementarias en polimicrogiria, pero ante los esperanzadores resultados obtenidos, se hace necesario realizar estudios con muestras mayores para poder obtener resultados representativos y establecer un protocolo sólido y validado.

Palabras Clave: musicoterapia, polimicrogiria, estimulación, parálisis cerebral.

\section{Music therapy in polymicrogyria. An intervention proposal}

ABSTRACT This study proposes a protocol for a music therapy intervention for patients suffering from polymicrogyria. This low prevalence disease is caused by an incorrect folding pathway on the cerebral cortex. After having done a review, some necessities accessible with music therapy and some therapic goals have been established. These goals have determined the activities design. After having developed the protocol, it has been undertaken with a 13 years old patient suffering from polymicrogyria. The results obtained have been positive, because music therapy has increased her attention, decreased her heart rate and stimulated her movement capacity. The study also has valorized the previous knowledge about music therapy of the patient's parents using a test. It has shown that music therapy is not considered as an official complementary therapy in polymicrogyria. With these results, it is necessary to gather higher sample so that more representative studies and validated protocols can be done.

Keywords: music therapy, polymicrogyria, stimulation, cerebral palsy.

Sumario: 1. Introducción 2. Marco teórico 3. Marco metodológico 4. Resultados 5. Discusión 6. Conclusiones 7. Referencias

Cómo citar. Ortega, R. (2019). Musicoterapia en Polimicrogiria. Una propuesta de intervención. Revista de Investigación en Musicoterapia, 3,131-147 https://doi.org/10.15366/rim2019.3.008

\footnotetext{
${ }^{1}$ Universidad Internacional de La Rioja. raqueloril@hotmail.com
} 


\section{Introducción}

Este trabajo tiene como objetivo principal desarrollar una propuesta de intervención musicoterapéutica para pacientes con una patología neurológica denominada polimicrogiria, una

enfermedad de baja prevalencia que pertenece a las denominadas Enfermedades Raras. Oficialmente una enfermedad es considerada rara cuando afecta a menos de 1 por cada 2000 ciudadanos (Portal sobre Enfermedades Raras y Medicamentos Huérfanos). Hablando en cifras generales, la Federación Española de Enfermedades Raras (FEDER), apunta que entre el 6 y el $8 \%$ de la población mundial padece alguna de estas enfermedades (datos de 2017 tras la actualización del estudio ENSERio). Y es que, tal y como se afirma en la Guía de Apoyo Psicológico para Enfermedades Raras de la FEDER, en este tipo de patologías se produce el fenómeno conocido como "Paradoja de la Rareza": si bien cada enfermedad por separado presenta una prevalencia muy baja, todas en su conjunto suponen un gran número de personas afectadas.

Entre los problemas a los que se enfrentan los pacientes con este tipo de patologías se encuentran la dificultad de diagnóstico y la falta de tratamiento. De hecho la FEDER estima que el $42,68 \%$ de ellos, no dispone de un tratamiento adecuado. Esta falta de tratamientos establecidos, pone de manifiesto que la realización de este trabajo puede tener gran interés para los pacientes con polimicrogiria. Por otro lado, esta patología se caracteriza por una alteración en la estructura de los pliegues del córtex cerebral que se acompaña por diversas manifestaciones que pueden ser abordadas y beneficiarse a través de la musicoterapia

Por todo ello, colaborar y ofrecer una nueva herramienta de tratamiento a través de la musicoterapia para esta patología, supone una gran motivación profesional como musicoterapeuta y además, constituye un instrumento de utilidad para el abordaje de la polimicrogiria.

\section{JUSTIFICACIÓN Y PROBLEMA}

Como se ha mencionado, la polimicrogiria es una enfermedad clasificada dentro de las Enfermedades Raras, lo cual puede justificar que no se hayan encontrado estudios previos del empleo de la musicoterapia en pacientes con esta patología debido a su baja prevalencia. Este hecho pone de manifiesto la importancia del presente trabajo, ya que puede aportar nuevos datos a la comunidad científica así como una herramienta de tratamiento para los pacientes con esta patología.

\section{OBJETIVOS GENERALES Y ESPECÍFICOS}

El trabajo cuenta con 2 objetivos generales: desarrollar una propuesta de intervención musicoterapéutica para pacientes afectados por una enfermedad neurológica denominada polimicrogiria, y estudiar en un caso concreto la respuesta a dicha propuesta. Los objetivos específicos y procedimientos que se concretan para ello son:

- Establecer las características de la polimicrogiria 
- Estudiar los antecedentes sobre el empleo de la musicoterapia en pacientes con polimicrogiria

- Determinar unas necesidades de este colectivo que puedan abordarse desde el punto de vista musicoterapéutico

- Evaluar la puesta en práctica de la intervención planteada para valorar su idoneidad

- Conocer la opinión de los padres sobre la musicoterapia como herramienta en la polimicrogiria

- Extraer conclusiones que argumenten el empleo de la musicoterapia en esta patología

\section{Marco Teórico}

\section{Polimicrogiria}

La polimicrogiria es un tipo de malformación del desarrollo cortical que cursa con una laminación anormal de la corteza cerebral y con un patrón inusual de sus pliegues, de manera que la totalidad o parte de la superficie cortical está cubierta de múltiples y pequeñas circunvalaciones o plegamientos El mecanismo por el que tiene lugar es un error en la migración neuronal, fenómeno que sucede durante el desarrollo fetal al viajar las neuronas desde el tubo neural (estructura embrionaria precursora del sistema nervioso) hasta su lugar definitivo (Leventer et al., 2010).

La etiología de esta enfermedad no está todavía del todo esclarecida, pudiendo ser de naturaleza exógena o genéticamente determinada. En el caso de la causa exógena, la infección intrauterina por citomegalovirus podría ser la desencadenante de un importante número de los casos. También puede ser producida por fenómenos de isquemia intrauterina (Barkovich, 2010). Desde el punto de vista genético cada vez se conocen más genes implicados en el desarrollo de esta patología, siendo el más importante es el síndrome de microdeleción 22q11.2 (Flotats-Bastardas et al., 2012).

Si bien se desconoce su prevalencia exacta, se sabe que es una enfermedad poco común, y por ello aparece recogida en el Portal Oficial de Enfermedades Raras y Medicamentos Huérfanos (en inglés, ORPHANET) con el código 35981.

En cuanto a su diagnóstico, se realiza mediante estudios anatomopatológicos del tejido cerebral. Sin embargo, gracias al desarrollo de las técnicas de imagen, y más concretamente al uso de la resonancia magnética $(\mathrm{RMN})$ se ha hecho posible el diagnóstico de la polimicrogiria sin necesidad de tomar muestras cerebrales (Flotats-Bastardas et al., 2012). A continuación se presentan dos imágenes de RMN. Al compararlas, puede observarse cómo la corteza del cerebro con polimicrogiria presenta numerosos e irregulares pliegues, como si se tratase de un trazo dibujado por una mano temblorosa. Por el contrario el cerebro sano, presenta menor número de pliegues y mucho más regulares. Es precisamente ese excesivo número de pliegues o giros el que da nombre a esta patología: poli-muchos, micro-pequeños, giria-gir 


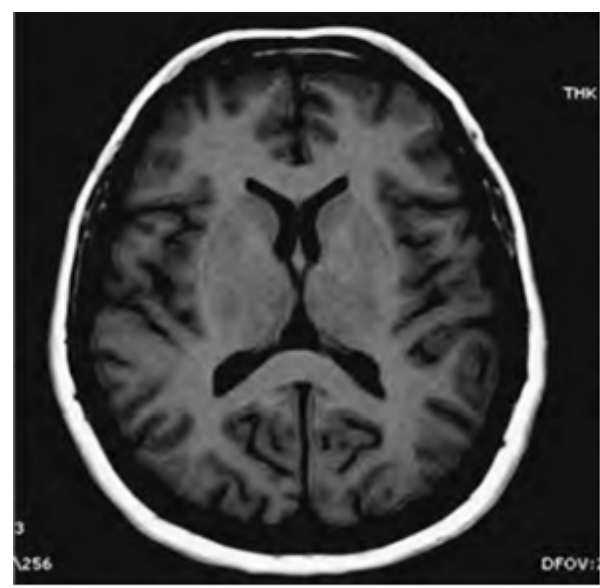

Figura 2: Corte axial de cerebro sano. Imagen obtenida por RMN

(Rivera et al., 2011)

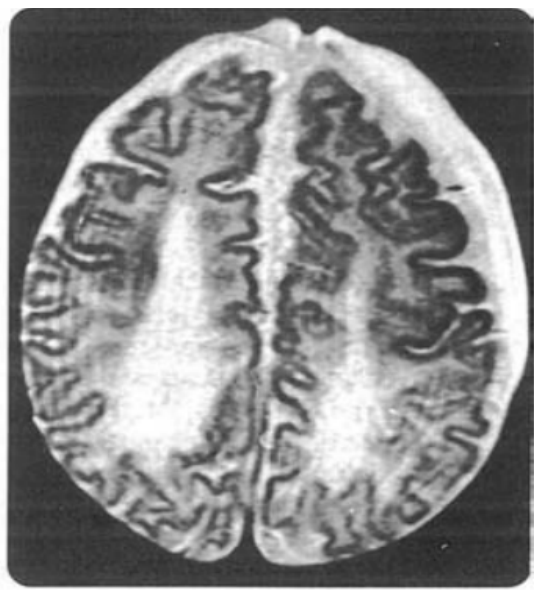

Figura 1: Giros anormales en

corte axial de cerebro con

polimicrogiria. Imagen obtenida por

RMN (Vélez-Domínguez, 1998)

En cuanto a sus manifestaciones clínicas, pueden presentarse cuadros muy diversos, pero los signos más importantes son: Discapacidad intelectual o Retraso Global del desarrollo, epilepsia, trastorno oro-motor, parálisis pseudolobular, hemi o tetraparesia y alteraciones neurosensoriales (hipoacusia, ceguera) (Flotats-Bastardas et al., 2012).

\section{ANTECEDENTES DEL EMPLEO DE LA MUSICOTERAPIA EN LA POLIMICROGIRIA}

No se han encontrado publicaciones de estudios de la musicoterapia en pacientes con polimicrogiria. Como ya se ha indicado se trata de una enfermedad de muy baja prevalencia, lo cual obstaculiza la realización de estudios de cualquier índole por la dificultad de reunir una muestra suficientemente grande que permita emitir resultados representativos. Asimismo, la polimicrogiria y en general las enfermedades raras, presentan una gran variabilidad de sintomatología, por lo que también resulta complicado unificar a las muestras de estudio (Federación nacional de Enfermedades Raras).

Por lo tanto la primera conclusión y quizás la más importante que se obtiene al analizar el estado de la cuestión es la carencia de estudios que corroboren que el empleo de la musicoterapia puede resultar beneficioso para estos pacientes. Ante la falta de medios, se ha optado por buscar evidencia científica del empleo de la musicoterapia en aquellos cuadros clínicos más importantes de la polimicrogiria: la parálisis cerebral, la epilepsia y el retraso global del desarrollo.

\section{Musicoterapia en parálisis cerebral}

El empleo de la música para la parálisis cerebral ha sido estudiado desde hace varias décadas. El estudio de Schneider en 1957 y el de Lathom y colaboradores en 1945, concluyeron que la música con ritmo rápido, volumen elevado y líneas melódicas con saltos controlaba a los niños atetósicos (aquellos con movimientos involuntarios lentos), mientras que la música de ritmo lento, melodías sin saltos, volúmenes bajos... beneficiaba a los niños con cuadros 
espásticos (aquellos con movimientos exagerados y reflejos). (Schneider, 1957, citado en Poch, 2011, p243 y Lathom, 1945, citado en Poch, 2011, p243).

Estudios más recientes muestran resultados muy positivos:

En 2016 se realizó una revisión sistemática sobre el empleo de la musicoterapia activa en pacientes con parálisis cerebral, es decir aquélla que implica que el paciente participe en la creación de la música. Concluyeron que la musicoterapia es una estrategia muy beneficiosa en potenciar los cambios de neuroplasticidad así como las interacciones sensoriomotoras (Alves-Pinto et al., 2016).

Por otro lado, en 2018 Marrades-Caballero y colaboradores llevaron a cabo un estudio randomizado con 18 niños entre 4 y 16 años que padecían parálisis cerebral severa. Tras 16 semanas de sesiones de musicoterapia neurológica aplicada al grupo intervención concluyeron que la musicoterapia es una herramienta muy positiva para la mejora en la funcionalidad de estos pacientes. (Marrades-Caballero et al., 2018).

\section{Musicoterapia en epilepsia}

El empleo de la musicoterapia en la epilepsia ha sido menos estudiado que en la parálisis cerebral. Serafina Poch en su Compendio de Musicoterapia, defiende que la música puede emplearse para el tratamiento de los síntomas psicológicos asociados a la epilepsia tales como irritabilidad, cambios de humor o angustia por la incertidumbre de la llegada de un nuevo episodio principalmente. Sin embargo, no hace hincapié en el control de las crisis epilépticas a través de la musicoterapia. (Poch, 2011).

Posteriormente, se ha llevado a cabo una revisión sistemática sobre el efecto de la música de Mozart en el control de las convulsiones y crisis epilépticas. Se revisaron 7 estudios y se concluyó que la música de Mozart resulta beneficiosa en el control de la epilepsia, no solo en tratamiento combinado con fármacos antiepilépticos, sino también en epilepsias resistentes a fármacos y a cirugía como tratamiento único (Brackney, D.E., Brooks J.L, 2018).

\section{Musicoterapia en retraso del desarrollo}

El retraso global del desarrollo se define como "una alteración grave y generalizada de varias áreas del desarrollo; concretamente, la interacción social, la conducta y la comunicación" (Martín-Luengo, 2010, p.64). Existen diversos estudios que demuestran que la musicoterapia resulta beneficiosa en enfermedades con esta manifestación.

Ya en 1978, la National Association for Music Therapy, mostró un estudio de Lathom que determinó cómo la musicoterapia ayuda en cuadros de retraso global contribuyendo a: aumentar las habilidades motrices, favorecer el contacto visual con el interlocutor, desarrollar la coordinación, ayudar al lenguaje mediante el canto y mejorar la discriminación auditiva (Lathom, 1978).

Acercándonos a nuestros días, en 2011, Pineda y Pérez llevaron a cabo un estudio con 18 niños con Síndrome de Down. Después de 9 meses con sesiones de musicoterapia, concluyeron que mejoraron aspectos del lenguaje, de socialización y de aprendizaje. Asimismo determinaron que es una herramienta muy positiva para mejorar su calidad de vida (Pineda y Pérez, 2011).

\section{Marco Metodológico}

El procedimiento que se ha llevado a cabo para cumplir los objetivos planteados se resume en la siguiente tabla. 
Tabla 1: Procedimiento metodológico (Elaboración propia)

\begin{tabular}{llrlll}
\hline Iniciación & Planificación & & Implementación & \multicolumn{2}{l}{ Evaluación } \\
\hline Búsqueda bibliográfica & Análisis de & las & Se testa la propuesta & Se analizan los \\
con PubMed, Google & necesidades & y & con una paciente con & resultados y se \\
Scholar, Biblioteca & diseño de & la & polimicrogiria & extraen conclusiones \\
UNIR y Dialnet & intervención & & & & \\
\hline
\end{tabular}

\section{Propuesta de INTERVEnCión}

\section{POBLACión Y MUESTRA}

La población sobre la que se desea aplicar la intervención que se propone en este trabajo es el conjunto de pacientes que sufren polimicrogiria. Tal y como se ha mencionado, por tratarse de una enfermedad rara de baja prevalencia y alta variabilidad resulta muy complejo reunir muestras representativas.

En este caso, la propuesta se testó sobre una muestra seleccionada de forma intencionada, es decir, sin ningún procedimiento aleatorizado de muestreo. Se trata de S.H., una niña de 13 meses, cuya causa de afección por esta enfermedad ha sido la infección intrauterina por citomegalovirus. Si bien se trata de una muestra mínima, permite realizar una evaluación real de la propuesta planteada, sin dejar el trabajo como algo meramente teórico. S.H nació con polimicrogiria bilateral, es decir con afectación de los dos hemisferios con las anomalías en la corteza cerebral que se han mencionado en apartados anteriores.

\section{ANÁLISIS DE NECESIDADES}

Una vez se ha descrito brevemente el cuadro que presenta la paciente, resulta fundamental establecer una serie de necesidades que puedan ser abordadas desde el punto de vista musicoterapéutico. Del análisis de las características de la paciente, se derivan una serie de puntos que pueden trabajarse en sesiones de musicoterapia a saber:

- Desarrollo de la intencionalidad comunicativa

- Mantenimiento de la atención

- Potenciación del control motriz general

- Estimulación de la musculatura facial, que está comenzando a despertar su actividad.

\section{OBJETIVOS TERAPÉUTICOS}

Teniendo en cuenta las necesidades anteriormente mencionadas, se establecen los siguientes objetivos terapéuticos:

- Aumentar el número de sonrisas que produce durante la sesión: con ello se favorece su intencionalidad comunicativa y se trabaja la musculatura facial 
- Disminuir la frecuencia cardiaca entre el comienzo de la sesión y el final: con ello se facilita que se encuentre más calmada y por tanto más atenta de manera mantenida

- Aumentar el tiempo que mantiene la mirada a su madre en una de las actividades de la sesión: con ello se estimula el trabajo de su atención mantenida

- Aumentar el número de veces que consigue coger el sonajero cuando se le ofrece: con ello se potencia el control motriz de su mano

- Aumentar el tiempo que agita el sonajero: de esta manera aumenta el control motriz del brazo

\section{METODOLOGÍA DE LA INTERVENCIÓN}

El diseño de las actividades de cada sesión, se ha basado en el propuesto por MateosHernández en 2004, donde divide cada sesión en las siguientes siete fases (MateosHernández, 2004, p. 185):

- Caldeamiento

- Motivación para la sesión

- Actividades sonoro musicales de activación corporal consciente

- Actividades sonoro musicales de desarrollo perceptivo

- Actividades sonoro musicales de relación con el otro

- Actividades sonoro musicales de representación y simbolización

- Actividades de despedida

Antes de proceder a desarrollar cada una de las actividades, se hace necesario mencionar la importancia de que los progenitores participen en las sesiones de musicoterapia con bebés. Existen diversos estudios que así lo avalan (Martin, 2018).

La estructura planteada para las sesiones sería:

a) Llegada, saludo y medida de la frecuencia cardiaca antes de comenzar

b) Actividades sonoro musicales de bienvenida: Se comienza con una canción de bienvenida en la que la madre y la musicoterapeuta cantan, y esta última además acompaña armónicamente. Es muy breve, de 2 minutos aproximadamente. Para ello, se van intercalando los acordes I-IV únicamente tocados con el arpa, tomando como referencia la intervención de María Jesús del Olmo con neonatos de la UCI (Del Olmo, 2009), quien mantuvo esta secuencia en todas las intervenciones de bienvenida. Tal y como ella explica: "estas dos funciones armónicas constituidas por los acordes de tónica y subdominante, no producen ninguna tensión ni disonancia”. (Rueda, 1994, citado en Del

Olmo, 2009, p. 70). Con esta actividad se pretende únicamente iniciar a la paciente en la Revista de Investigación en Musicoterapia, 3, 2019, pp.131-147 
sesión. Es importante que la madre cante junto con la musicoterapeuta, para que la paciente se sienta más arropada y el ambiente le resulte lo más familiar posible.

c) Actividades sonoro musicales de motivación: en este caso, y siguiendo las indicaciones de Serafina Poch (Poch, 2011), se emplean músicas que cambien el tempo, el tono, la intensidad... de manera que resulten especialmente estimulantes. Se busca con ellas potenciar su sonrisa (trabajo de los músculos faciales y de la intención comunicativa) y en general fomentar que se divierta (movimiento del maxi-cosi, de los pies...) En este caso, se ha recurrido a la música grabada, para poder observar con más detenimiento sus respuestas y además para que los recursos musicales sean de mayor envergadura. La pieza elegida para esta actividad es: La Danza Rusa del Cascanueces de Tchaikovsky (Suite "E1 Cascanueces Op.71a).

d) Actividades sonoro musicales de actividad corporal: en este caso se trabaja el aspecto motriz. La actividad consiste en lo siguiente: en primer lugar, se ofrece a la niña su sonajero para que lo coja y se trabaje así tanto la atención como la capacidad motriz de su mano. Se le ofrece un total de 3 veces por sesión y si en ninguna de las 3 logra cogerlo se le coloca en la mano. Posteriormente la madre, que dispone de una maraca, la agita delante de la niña marcando el ritmo de la pieza. La pieza musical escogida para esta actividad es la sección de trompeta de la Marcha Triunfal de Aída (G. Verdi) una pieza rítmica, tal y como propone Serafina Poch en su Compendio de Musicoterapia. Además, la musicoterapeuta marcará el ritmo con un pandero.

Para el diseño de esta actividad se han tomado como referencia dos aspectos fundamentales: por un lado, las neuronas espejo. Según Emilio García, las neuronas espejo son:

Un tipo particular de neuronas que se activan cuando un individuo realiza una acción, pero también cuando él observa una acción similar realizada por otro individuo. Las neuronas espejo forman parte de un sistema de redes neuronales que posibilita la percepción-ejecución-intención. La simple observación de movimientos de la mano, pie o boca activa las mismas regiones específicas de la corteza motora, como si el observador estuviera realizando esos mismos movimientos. (García, 2008).

Conociendo la existencia de estas neuronas, el hecho de que la madre de la paciente realice el movimiento de agitar la maraca delante de ella, invitará a que la niña realice ese mismo movimiento con su sonajero.

En segundo lugar, en la relación que existe entre el sistema auditivo y el sistema motor: en 1976, Rossignol y Melvill-Jones hallaron que existen vías audio motoras que viajan desde las zonas activadas por el estímulo sonoro, hasta las motoneuronas de la médula espinal, donde incrementan y regulan la excitabilidad de las mismas (Rossignol y MelvillJones, 1976). Este descubrimiento, ha permitido que la música haya sido utilizada en el 
trabajo del control muscular en niños con trastornos neuromusculares así como en rehabilitaciones de Parkinson dentro del marco del modelo conductista de musicoterapia.

e) Actividades sonoro musicales de desarrollo perceptivo: con estas actividades trabaja la atención mantenida. Para ello, ha contado nuevamente con la participación de la madre. La actividad consiste en lo siguiente: la madre canta cerca de la niña acompañada por la musicoterapeuta con el arpa. Durante el primer minuto, la madre canta la canción quieta y muy cerca de la cara de la paciente. Posteriormente, se mueve por el espacio para que la niña le siga con la mirada.

f) Actividades de despedida: a través de esta actividad, se pretende indicar el fin de la sesión y conseguir un estado de relajación en el bebé contribuyendo a la disminución de su frecuencia cardiaca. Para ello, se ha escogido una pieza que guste a la madre, y se ha nanificado, es decir se ha convertido en compás ternario y se ha bajado su tempo. Para esta sesión en concreto, se preguntó a la madre por una canción que le gustase y tuviera significado para ella, escogiendo Who would imagine a King, de Whitney Houston (banda sonora original de la película The preacher's wife, 1996).

La madre acompañada por la musicoterapeuta al arpa, canta esa canción a la paciente. El hecho de realizar actividades con el canto de la madre favorece la relajación de los bebés, ya que se ha comprobado que disminuye sus niveles de la hormona de estrés por excelencia, el cortisol. (Threub, 2003, citado en Del Olmo, 2009, p 69). Además el empleo del ritmo ternario, y de música de interés para los padres, se basa en el método musicoterapéutico del RBL (Rythm, Breath, Lullaby). Se trata de un método musicoterapéutico que surge para el trabajo con bebés prematuros en la UCI neonatal y que ha demostrado mejorar diversos parámetros fisiológicos tales como la frecuencia respiratoria, la frecuencia cardiaca, el reflejo de succión, la ganancia de peso... empleando sonoridades que imitan los sonidos intrauterinos mediante el Ocean drum y el Tone box. Así lo demuestra la revisión bibliográfica de literatura sobre musicoterapia en recién nacidos llevada a cabo en 2018 (Martin, 2018). En este caso, no se van a emplear los mencionados instrumentos porque no se trata de un procedimiento de RBL como tal, sino que simplemente toma de este método algunas características como el escoger música de interés para los padres y nanificarla, lo cual tiene muchos beneficios para conseguir una relajación del bebé y una potenciación del vínculo materno filial.

g) Medición de la frecuencia cardiaca: se espera 1 minuto tras haber terminado la última actividad de la sesión y se mide la frecuencia cardiaca.

\section{TEMPORALIZACIÓN}

Se han llevado a cabo 4 sesiones semanales. Para determinar la duración, se han tomado dos referencias: por un lado lo indicado por Serafina Poch en su Compendio de Musicoterapia (Poch, 2011), que son 30 minutos; por otro, el estudio llevado a cabo con bebés de 6 meses 
por Sandra Threub en 2003, con una duración de 15 minutos (Threub, 2003). En este caso, el tiempo de las sesiones se estima en 20 minutos. Antes de la primera sesión, se realizará una reunión con la madre, para explicarle en qué van a consistir las sesiones, resolver todas sus inquietudes y explicarle cómo ella también será partícipe en el desarrollo de las mismas. En esta reunión, además, entregará y firmará el consentimiento informado, tal y como recoge el Código Ético para los Musicoterapeutas Profesionales en España (CEMPE) en su artículo 6.

\section{RECURSOS}

Los recursos necesarios para llevar a cabo la intervención son:

Recursos humanos: el musicoterapeuta, la madre y la paciente.

Recursos materiales: espacio (las sesiones se realizan en el salón de la casa en la que vive la paciente), dispositivo de reproducción de música, cronómetro, maraca, sonajero (para que la niña estuviese familiarizada con el objeto, las actividades se han realizado con su propio sonajero), arpa (ha sido el instrumento polifónico utilizado para las sesiones. Por su mejor y más fácil manejo se ha utilizado un arpa celta).

\section{HERRAMIENTAS DE EVALUACIÓN}

Las variables empleadas para valorar los resultados de esta intervención, se han recogido en una hoja de observación durante cada una de las sesiones. Dichos parámetros son:

- Variables fisiológicas: se ha utilizado la frecuencia cardiaca. Para medirla, se ha tomado como referencia el protocolo seguido por María Jesús Del Olmo en su tesis doctoral (Del Olmo, 2009). Se ha medido la frecuencia cardiaca 5 minutos antes de comenzar la sesión, a los 5 minutos de haber comenzado la sesión, y 1 minuto después de haber terminado la sesión. Para la toma de la frecuencia cardiaca, y ante la ausencia de monitor, se ha tomado el pulso en la arteria radial con los dedos índice y corazón durante 15 segundos y posteriormente se ha multiplicado el valor por cuatro obteniendo así el número de latidos por minuto.

- Variables psicológicas: En este caso se han utilizado dos parámetros psicológicos, a saber: la sonrisa y la mirada atenta. Ambos parámetros también fueron analizados en la tesis doctoral de Del Olmo (2010) dentro de los parámetros psicológicos. Esta autora considera que tanto la sonrisa como la mirada atenta, son dos signos que el bebé utiliza para comunicarse con el adulto y que cuando se acompañan de música, la interacción tiene consecuencias terapéuticas (Del Olmo, 2009, p 112). En el caso de la sonrisa, se ha cuantificado de forma discreta durante toda la sesión anotando en la hoja de observación el número de veces que la paciente sonríe. Se ha tenido en cuenta también el momento en el que lo hace, para poder después establecer algunas conclusiones al respecto. En cuanto a la medición de la mirada atenta, se ha cronometrado el tiempo que la paciente mantiene la 
mirada a su madre en una de las actividades de la sesión que se explicará más adelante. En este caso se ha anotado el tiempo en segundos que S.H. mantiene la mirada en dicha actividad. Posteriormente se ha calculado el porcentaje de tiempo que supone respecto al total de la actividad (90 segundos), para así representar mejor la información.

- Variables físicas: este tipo de variables están relacionadas con el movimiento. Como el trabajo motriz es uno de los aspectos más importantes en esta patología, se han tenido especialmente en cuenta. Por un lado, se han anotado de manera dicotómica (Sí-No) si consigue coger el sonajero cuando se le ofrece y si lo consigue en el intento número 1, 2 ó 3. El resultado se ha recogido en la hoja de observación de la sesión. Por otro lado, se ha cuantificado el tiempo que lo mantiene sujeto, y el tiempo durante el cual lo agita.

Además de estas variables, se ha medido la percepción de la familia sobre la musicoterapia como posible herramienta de tratamiento para la polimicrogiria. Para ello, se ha pasado a los padres un cuestionario vía Google Drive con 19 ítems al respecto.

Para la toma de todos los datos se emplea una hoja de observación creada ad hoc para esta intervención.

\section{Resultados}

\section{RESULTADOS DEL CUESTIONARIO A LA MADRE DE LA PACIENTE}

En este apartado se plasman las respuestas que se han obtenido del cuestionario realizado a la madre de la paciente. Las respuestas de los 19 ítems se recogen en la siguiente tabla:

Tabla 2: Respuestas del cuestionario realizado a la madre de la paciente (Elaboración propia).

\begin{tabular}{|c|c|}
\hline Pregunta & Respuesta \\
\hline 1. Edad & 42 \\
\hline 2. Nivel de estudios & Universitarios \\
\hline 3. Sabe algo sobre Musicoterapia & Sí \\
\hline 4. ¿Qué función tiene la Musicoterapia? & $\begin{array}{l}\text { Mejorar la calidad de vida y contribuir al } \\
\text { bienestar }\end{array}$ \\
\hline 5. ¿Quién debe ejercer la Musicoterapia? & Musicoterapeuta formado específicamente \\
\hline 6. En las sesiones de Musicoterapia & $\begin{array}{l}\text { Puede emplearse tanto música grabada como } \\
\text { música en vivo }\end{array}$ \\
\hline 7. En las sesiones de Musicoterapia & Pueden participar el paciente y algunos \\
\hline
\end{tabular}


8. La Musicoterapia y la Polimicrogiria

9. La participación activa de los padres en sesiones con bebés

Resulta positivo

10. La música puede conseguir estimular las neuronas del movimiento sin necesidad No lo sé de ejecutarlo

11. ¿Cree que la Musicoterapia debería estar más extendida como tratamiento de Sí patologías como la Polimicrogiria?

12. ¿Cree que la población en general está suficientemente informada sobre esta No disciplina?

13. ¿Le hubiera gustado que el hospital ofreciese sesiones de Musicoterapia Sí dentro del marco de la rehabilitación?

14. ¿Cree que esto podría ocurrir en el futuro?

15. ¿Cree que su hijo/a podría mejorar algún aspecto de su enfermedad mediante las Sí sesiones de Musicoterapia?

16. ¿En qué cree que van a consistir las sesiones de Musicoterapia en Polimicrogiria?

Supongo que inicialmente en que la niña escuche algún tipo de música y observar de qué manera influye ese estímulo en su comportamiento

17. ¿A través de qué medio obtuvo la información que conoce sobre Musicoterapia?

Otros (Ni familia o amigos ni campañas de hospital ni medios de comunicación)

18. ¿Sabe si alguna institución de su comunidad realiza charlas informativas No sobre Musicoterapia?

19. ¿Recomendaría esta disciplina a otras familias con situaciones similares a la Sí suya?

\section{RESULTADOS DE LA INTERVENCIÓN}

En primer lugar, se muestran los datos de carácter cualitativo, para proceder con los gráficos y tablas de carácter más cuantitativo, que se han elaborado con la herramienta Excel. 


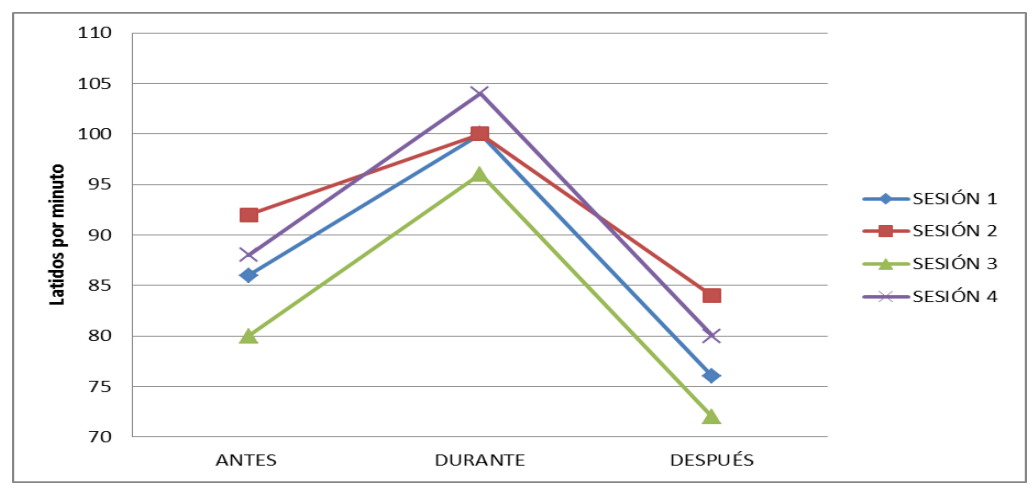

Figura 3: Frecuencia cardiaca antes, durante y después de la intervención (Elaboración propia)

Tabla 3: Valores de frecuencia cardiaca antes, durante y después de la intervención (Elaboración propia)

\begin{tabular}{llll} 
& FC media antes & FC media durante & FC media después \\
\hline SESIÓN 1 & 86 & 100 & 76 \\
\hline SESIÓN 2 & 92 & 100 & 84 \\
\hline SESIÓN 3 & 80 & 96 & 72 \\
\hline SESIÓN 4 & 88 & 104 & 80 \\
\hline MEDIA & $\mathbf{8 6 . 5}$ & $\mathbf{1 0 0}$ & $\mathbf{7 8}$ \\
\hline
\end{tabular}

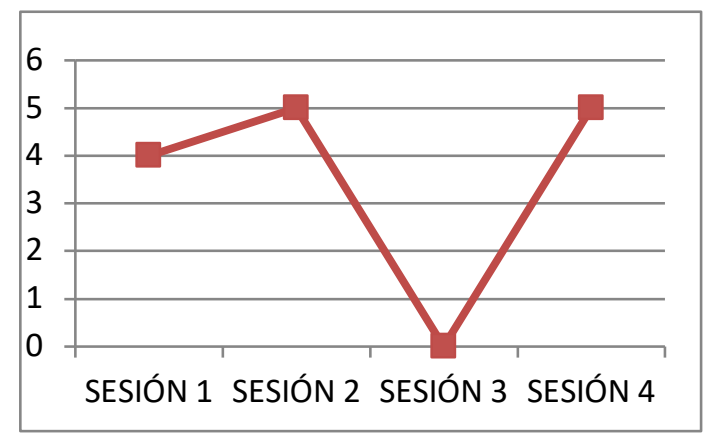

Figura 5: porcentaje de tiempo que mantiene la mirada (Elaboración propia)

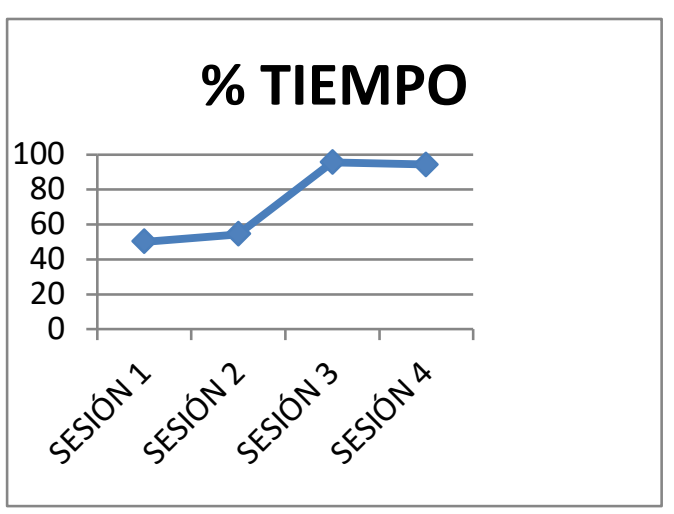

Figura 4 Número de sonrisas en cada sesión (Elaboración propia 
Tabla 4: Media del número de intentos necesarios para coger el sonajero (Elaboración propia)

\begin{tabular}{cccc}
\hline $\begin{array}{c}\mathbf{N}^{0} \text { intentos en } \\
\text { sesión 1 }\end{array}$ & $\begin{array}{c}\mathbf{N}^{0} \text { intentos en } \\
\text { sesión 2 }\end{array}$ & $\begin{array}{c}\mathbf{N}^{\mathbf{0}} \text { intentos en } \\
\text { sesión 3 }\end{array}$ & Media de intentos \\
\hline 1 & 1 & 1 & 1 \\
\hline
\end{tabular}

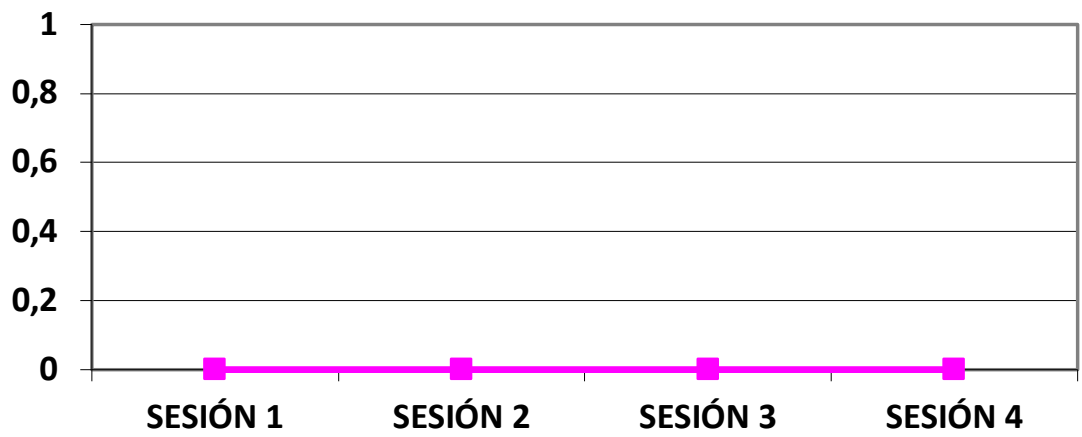

Figura 6 : Tiempo durante el cual agita el sonajero (Elaboración propia)

Tabla 5: Valoración (sobre 10 ptos) de los aspectos experienciales durante las sesiones (Elaboración propia)

\begin{tabular}{llllll}
\hline Aspecto & $\mathbf{1}^{\mathbf{a}}$ sesión & $\mathbf{2}^{\mathbf{a}}$ sesión & $\mathbf{3}^{\mathbf{a}}$ sesión & $\mathbf{4}^{\mathbf{a}}$ sesión & Media \\
\hline Interacción & 10 & 10 & 10 & 10 & 10 \\
\hline Disfruta & 10 & 10 & 10 & 10 & 10 \\
\hline Participación & 10 & 10 & 10 & 10 & 10 \\
\hline Displacer & 0 & 0 & 0 & 0 & 0 \\
\hline Rechazo & 2 & 0 & 0 & 0 & 0.5 \\
\hline
\end{tabular}

\section{Discusión}

La presente discusión, se centra en los resultados más relevantes obtenidos de la puesta en práctica de dicha intervención, si bien estos resultados no pueden considerarse totalmente válidos al no haberse realizado la práctica en las condiciones necesarias.

Por un lado, el cuestionario realizado a la madre de la paciente, ha servido para poder considerar la musicoterapia dentro del tratamiento integral de la polimicrogiria. Tal y como se observa en los ítems 13, 15 y 19 (Tabla 3), la madre consideraría la musicoterapia como una buena opción de tratamiento para la polimicrogiria, y le hubiera gustado que el hospital le hubiera propuesto alguna actividad de este tipo. Asimismo, pueden extraerse algunas conclusiones sobre el conocimiento e información previa que las familias tienen sobre la musicoterapia. La madre de la paciente S.H., sí que conoce ciertos aspectos sobre esta disciplina (ítems 3-9, Tabla 3), sin embargo desconoce otros. Estos resultados pueden compararse con los obtenidos en el estudio de Pineda y Pérez (2011), en el que se preguntaba a padres de hijos con Síndrome de Down sobre sus conocimientos de la musicoterapia como Revista de Investigación en Musicoterapia, 3, 2019, pp.131-147 
posible tratamiento complementario. En este estudio se encuestó a 18 padres, y 13 de ellos (el $72,2 \%$ ) afirmaron no tener conocimientos sobre musicoterapia. Si bien sería necesario encuestar a más familias con hijos que padezcan polimicrogiria, resulta interesante comprobar que otros estudios se ha optado también por realizar una encuesta previa a los padres para determinar qué conocimientos tienen sobre la terapia que se va a aplicar a sus hijos.

En cuanto a los datos obtenidos de la intervención, parece ser que la musicoterapia muestra resultados positivos.

En primer lugar respecto a la frecuencia cardiaca, los resultados muestran una tendencia bastante clara: las actividades de estimulación generan en la paciente un aumento de sus latidos por minuto. Estos resultados tienen sentido, ya que en las actividades iniciales de la sesión se trabaja la estimulación, tanto motriz como cognitiva. Sin embargo, una vez terminada la sesión y habiendo realizado la actividad de relajación, la frecuencia cardiaca muestra un significativo descenso. Estos resultados concuerdan con los obtenidos en la Tesis Doctoral de Del Olmo, donde se concluyó que la frecuencia cardiaca de los bebés después de las sesiones de musicoterapia descendía de un modo estadísticamente significativo (Del Olmo, 2009). Si bien los resultados han mostrado un descenso de la frecuencia cardiaca en la paciente S.H., este beneficio no es específico para el tratamiento de la polimicrogiria, sino en general para los bebés que puedan encontrarse en cualquier situación de agitación.

Analizando el apartado de las sonrisas, esta medición se había incluido dentro de las variables psicológicas, ya que se trata de un signo facial que refleja emociones positivas. Si bien no puede definirse una tendencia ascendente clara, la paciente ha mostrado un número elevado de sonrisas durante las sesiones (según la madre le cuesta mucho sonreír y el hecho de que lo hiciera de 4 a 5 veces durante el tiempo que duraban las sesiones era muy llamativo). Este hecho no solo es signo de bienestar psicológico, sino que además, sirve como ejercicio de estimulación de la musculatura facial; hecho que podría enmarcarse dentro de una rehabilitación multidisciplinar. Estos resultados concuerdan con los obtenidos en un estudio randomizado donde se concluyó que la musicoterapia ayuda a ganar funcionalidad motora en los pacientes con parálisis cerebral (Marrades-Caballero et al., 2018).

En cuanto al aspecto atencional, las sesiones han supuesto un estímulo buenísimo. El tiempo durante el que ha mantenido la mirada en la actividad de desarrollo perceptivo, no solo ha sido elevado sino que ha mostrado una tendencia ascendente (Gráfica 3). También se hace necesario destacar cómo la pieza de La Danza Rusa de El Cascanueces de Tchaikovski, estimulaba su capacidad atencional. Esta pieza producía en S.H. una situación de alerta, buscando la fuente sonora con la mirada e incluso generando en ella una posición más erguida y atenta. El hecho de que esta pieza haya resultado tan positiva, puede tener que ver con la clasificación que realiza Serafina Poch en su Compendio de Musicoterapia. La pieza en cuestión sería por un lado música que incita al movimiento, por ser de carácter folklórico y bailable, y además música jocosa que resulta motivadora, por el hecho de tener cambios en la intensidad y saltos melódicos (Poch, 2011, p. 215-216). El ser una pieza estimulante en ambos sentidos puede ser la explicación de su efecto tan intenso sobre el aspecto atencional.

El trabajo del aspecto motriz ha sido uno de los más complicados. A la hora de coger su sonajero, la paciente ha sido capaz de cogerlo en el primer intento en todas las sesiones que se han llevado a cabo (Tabla 4). Sin embargo, no se ha podido agitar el sonajero pese a haber realizado el movimiento de la maraca por parte de la madre empleando la potencia de las neuronas espejo y una música altamente rítmica que pusiera en marcha sus motoneuronas (Gráfica 4). No obstante, en dos de las sesiones (sesión 3 y 4) la paciente, tras haber cogido el 
sonajero en el primer intento, consigue lanzar el sonajero. No es exactamente una agitación "tipo maraca", pero sí que supone un movimiento del brazo así como una actividad cognitiva. Esta herramienta de evaluación podría ser susceptible de cambio en futuras sesiones, y analizar no tanto si agita el sonajero, sino si consigue lanzarlo, y el número de lanzamientos que realiza en el tiempo que dure la pieza rítmica.

Por último el análisis de los aspectos experienciales, muestran que la paciente ha estado a gusto durante las sesiones.

\section{Conclusiones}

El presente estudio ha contribuido de manera eficaz al cumplimiento de los objetivos específicos planteados en su inicio.

En primer lugar, se han establecido las características de la polimicrogiria, concluyéndose que se trata de una enfermedad de muy baja prevalencia, enmarcada dentro de las llamadas Enfermedades Raras y que cursa con una estructura anómala de la corteza cerebral. Asimismo, el estudio también ha permitido estudiar los antecedentes en el empleo de la musicoterapia en la polimicrogiria, llegándose a la conclusión de que no se han publicado estudios al respecto.

En cuanto a la determinación de las necesidades de los pacientes con polimicrogiria que pueden abordarse mediante la musicoterapia, el estudio también ha resultado favorable. Tras haber estudiado las características de la patología, se ha concluido que estas necesidades son: el desarrollo de la intencionalidad comunicativa, el mantenimiento de la atención, la potenciación del control motriz y la estimulación de la musculatura facial. Si bien el objetivo del trabajo era establecer unas necesidades para la población con esta patología, siempre se deberán analizar en cada caso concreto. Con el planteamiento anterior, se ha podido valorar la propuesta diseñada en una paciente real. Si bien solamente se ha testado en un caso, y en unas condiciones no óptimas para la investigación, los resultados obtenidos parecen mostrarse positivos ya que mejoran su desarrollo de la musculatura facial, reducen su frecuencia cardiaca y estimulan su capacidad atencional.

Otro objetivo del trabajo era conocer la opinión de las familias con polimicrogiria sobre la idea de utilizar la musicoterapia en el global del tratamiento de estos pacientes, concluyéndose que los padres estarían a favor de incluirla.

\section{Referencias}

Alves-Pinto, A., Turova, V., Blumenstein, T., Lampe, R. (2016). The case for musical instrument training in cerebral palsy for neurorehabilitation. Neural plasticity, 2016, 1-9

Barkovich, A. J. (2010). Current concepts of polymicrogyria. Neuroradiology, 52(6), 479487.

Brackney, D. E., Brooks, J. L. (2018). Complementary and alternative medicine: The Mozart Effect on childhood epilepsy. A systematic review. The Journal of School Nursing, 34(1), 28-37.

Código Ético para Musicoterapeutas Profesionales en España (2011). Recuperado de: https://musicoterapeutas.wixsite.com/aemp/documentos (9/Julio/2019). 
Del Olmo, M. J. (2009). Musicoterapia con bebés de 0 a 6 meses en cuidados intensivos pediátricos. Tesis doctoral. Universidad Autónoma de Madrid.

Federación Nacional de Enfermedades Raras (FEDER). Recuperado de https://www.enfermedades-raras.org/ el 7/Abril/2019

Flotats-Bastardas, M., Sánchez-Montañez, A., Vázquez-Méndez, E., Ortega-Aznar, A., Boronat-Guerrero, S., Raspall-Chaure, M., Toro-Riera, M., Munell, F., MacayaRuiz, A., Roig-Quilis, M. (2012). Variabilidad clínica de la polimicrogiria: a propósito de 35 nuevos casos y revisión de la bibliografía. Revista Neurología, 55(6), 321-329.

García, E. (2008). Neuropsicología y educación. De las neuronas espejo a la teoría de la mente. Revista de psicología y educación, 1(3), 69-90.

Leventer, R. J., Jansen, A., Pilz, D. T., Stoodley, N., Marini, C., Dubeau, F., Malone, J., Mitchell, L.A., Mandelstam, S., Scheffer, I.E., Berkovic, S.F., Andermann, F., Andermann, E., Guerrini, R., Dobyns, W. B. (2010). Clinical and imaging heterogeneity of polymicrogyria: a study of 328 patients. Brain, 133(5), 14151427.

Marrades-Caballero, E., Santonja-Medina, C. S., Sanz-Mengibar, J. M., Santonja-Medina, F. (2018). Neurologic music therapy in upper-limb rehabilitation in children with severe bilateral cerebral palsy: a randomized controlled trial. European journal of physical and rehabilitation medicine, 54(6), 866-872.

Martín-Luengo, B. (2010). Musicoterapia aplicada a los trastornos generalizados del desarrollo. Educación y Futuro, 23, 62-80.

Martin, S. (2018). Music Therapy with Premature Infants and their Parents in the NICU Setting. . Expressive Therapies Capstone Theses, 39. Universidad de Lesley.

Mateos-Hernández. L.A. (2004). Actividades musicales para atender a la diversidad. Madrid. España: ICCE

Pineda Pérez, E., y Pérez Remón, Y. (2011). Musicoterapia aplicada a niños con síndrome de Down. Revista cubana de Pediatría, 83(2), 142-148.

Poch, S. (2011). Compendio de Musicoterapia I. Barcelona. España. Herder. (2a Edición).

Portal sobre Enfermedades Raras y Medicamentos Huérfanos (ORPHANET). Recuperado de https://www.orpha.net/consor/cgi-bin/index.php?lng=ES el 7/Abril/2019

Rivera D. M., Puentes S., Caballero L. (2011). Resonancia magnética cerebral: secuencias básicas e interpretación. Universidad Médica de Bogotá, 52 (3): 292-306.

Rossignol, S., Melvill-Jones, G. (1976). Audio-spinal influence in man studied by the Hreflex and its possible role on rhythmic movements synchronized to sound. Electroencephalography and Clinical Neurophysiology, 41 (1), 83- 92.

Threub, S. (2003). Maternal singing modulates infant arousal. Toronto: Phsycology of Music 31, (4) 365-375.

Vélez-Domínguez, L.C. (1998). Trastornos de migración neural. Gaceta Médica de México, 134(2), 207-215.

\section{Agradecimientos:}

A mi tutor Rafael Martín Castilla por su cercanía y ayuda incondicional.

A Ángela Valls Ventura-Oteiza por su participación desinteresada y entusiasta en el estudio. 